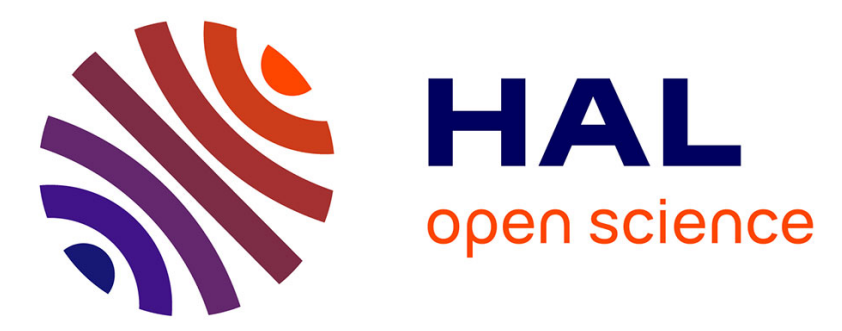

\title{
Exponential stabilization of boundary controlled port-Hamiltonian systems with dynamic feedback.
}

Héctor Estay, Yann Gorrec, Alessandro Macchelli, Hans Zwart

\section{To cite this version:}

Héctor Estay, Yann Gorrec, Alessandro Macchelli, Hans Zwart. Exponential stabilization of boundary controlled port-Hamiltonian systems with dynamic feedback.. IEEE Transactions on Automatic Control, 2014, 59 (10), pp.2849-2855. hal-01324673

\section{HAL Id: hal-01324673 \\ https://hal.science/hal-01324673}

Submitted on 1 Jun 2016

HAL is a multi-disciplinary open access archive for the deposit and dissemination of scientific research documents, whether they are published or not. The documents may come from teaching and research institutions in France or abroad, or from public or private research centers.
L'archive ouverte pluridisciplinaire HAL, est destinée au dépôt et à la diffusion de documents scientifiques de niveau recherche, publiés ou non, émanant des établissements d'enseignement et de recherche français ou étrangers, des laboratoires publics ou privés. 


\section{Exponential stabilization of boundary controlled port-Hamiltonian systems with dynamic feedback}

\author{
Héctor Ramírez*, Yann Le Gorrec*, Alessandro Macchelli ${ }^{\dagger}$ and \\ Hans Zwart ${ }^{\ddagger}$
}

\begin{abstract}
It is shown that a strictly-input passive linear finite dimensional controller exponentially stabilizes a large class of partial differential equations actuated at the boundary of a one dimensional spatial domain. This follows since the controller imposes exponential dissipation of the total energy. The result can by use for control synthesis and for the stability analysis of complex systems modeled by sets of coupled PDE's and ODE's. The result is specialized to port-Hamiltonian control systems and a simplified DNA-manipulation process is used to illustrate the result.
\end{abstract}

\section{INTRODUCTION}

Boundary control systems (BCS) [1]-[4] are a class of abstract systems that model partial differential equations (PDEs) with the control and the observations at the boundary of their spatial domain. A large class of physical systems may be modelled as BCS, and very powerful results on well-posedness and stability of solutions have been reported in recent works for BCS formulated using the framework of infinite dimensional port-Hamiltonian systems, [5] [9]. These results are promising since they provide constructive techniques for control design. More specifically, in [7] it has been shown that a clever choice of the boundary conditions (static feedback) renders the BCS exponentially stable, and in [6], [8] it has been shown that a power preserving interconnection with a finite dimensional Strictly Positive Real (SPR) linear system results in an asymptotically stable BCS on an extended state space. The SPR controller is used to assure the asymptotic stability, which is not straightforward to prove since it requires the application of LaSalle's invariance Theorem.

In this technical note, we show that a BCS with linear dynamic boundary control is exponentially stable provided that the controller is exponentially stable and strictly input passive. The closed-loop system is then an exponentially stable BCS on an extended state space. This follows from two key results. First, that the power preserving interconnection between BCS and SPR systems (and consequently between BCS and the class of controllers we consider) defines again a BCS on an extended space; and, second, that if the dissipated energy of the controller bounds the energy flow at the boundaries of the spatial domain the closed-loop BCS is exponentially stable. These results allow to elegantly, and quite easily, prove the exponential stability for a large class of linear controllers, in particular those defined by port-Hamiltonian control system.

The technical note is organized as follows. Section II introduces boundary controlled-port Hamiltonian systems and the definition of a class of BCS on an extended state space using dynamic boundary control. In Section III, a set of Lemmas derived from the definition of the finite dimensional control system are presented. Section IV presents the exponential stability proof of the closed-loop system.

*FEMTO-ST Institute, AS2M department, Université de FrancheComté/CNRS/ENSMM/UTBM, 24 rue Alain Savary, F-25000 Besancon, France. (e-mail: hector.ramirez@femto-st.fr, legorrec@femto-st.fr)

${ }^{\dagger}$ Department of Electrical, Electronic and Information Engineering "Guglielmo Marconi" (DEI), University of Bologna, viale del Risorgimento 2, 40136 Bologna, Italy. (e-mail: alessandro.macchelli@unibo.it)

$\ddagger$ University of Twente, Faculty of Electrical Engineering, Mathematics and Computer Science, Department of Applied Mathematics, P.O. Box 2177500 AE Enschede, The Netherlands. (e-mail: h.j.zwart@utwente.nl)

This work was supported by French ANR sponsored projects HAMECMOPSYS and Labex ACTION under reference codes ANR-11-BS03-0002 and ANR-11-LABX-01-01, respectively.
In Section V the control design is illustrated on a simplified DNAmanipulation process. Finally, Section VI provides some concluding remarks and comments on future work.

\section{BOUNDARY CONTROL SYSTEMS}

In the following, we briefly recall the main definitions of the BCS of interest. The reader is referred to [5], [7] and in particular to [8], [9] for further details. For comprehensive background on infinite dimensional linear system theory and functional analysis the reader is referred, respectively to [2]-[4] and [10]. We will follow the same notation as in [7], thus $M_{n}(\mathcal{H})$ denotes the space of square $n \times n$ matrices whose entries lie in the vector space $\mathcal{H}$. By $\langle\cdot, \cdot\rangle_{\mathbb{R}}$ we denote the inner product on $\mathbb{R}$ or $\mathbb{R}^{n}$, and $\langle\cdot, \cdot\rangle_{L_{2}}$, or simply $\langle\cdot, \cdot\rangle$ denotes the standard inner product on $L_{2}\left(a, b, \mathbb{R}^{n}\right)$. The Sobolev space of order $k$ is denoted by $H^{k}\left(a, b, \mathbb{R}^{n}\right)$. We say that a symmetric matrix is positive definite, in short $M>0$, if all its eigenvalues are positive, and positive semi-definite, in short $M \geq 0$ if its eigenvalues are nonnegative. A self-adjoint operator $L$ is coercive on an inner product space $X$ if there exists an $\epsilon>0$ such that $L \geq \epsilon I$.

The systems under study are described by the following PDE

$$
\left.\frac{\partial x}{\partial t}=P_{1} \frac{\partial}{\partial z}(\mathcal{L}(z) x)(t, z)\right)+\left(P_{0}-G_{0}\right) \mathcal{L}(z) x(t, z),
$$

$z \in(a, b)$, where $P_{1} \in M_{n}(\mathbb{R})$ is a non-singular symmetric matrix, $P_{0}=-P_{0}^{\top} \in M_{n}(\mathbb{R}), G_{0} \geq 0 \in M_{n}(\mathbb{R})$ and $x$ takes values in $\mathbb{R}^{n}$. Furthermore, $\mathcal{L}(\cdot) \in M_{n}\left(L_{2}(a, b)\right)$ is a continuously differentiable matrix-valued function satisfying for all $z \in(a, b), \mathcal{L}(z)=\mathcal{L}(z)^{\top}$ and $\mathcal{L}(z)>m I$, with $m>0$ independent from $z$.

For simplicity $\mathcal{L}(z) x(t, z)$ will be denoted by $(\mathcal{L} x)(t, z)$ and we shall use the notation $\mathcal{J}=\left(P_{1} \frac{\partial}{\partial z}+P_{0}\right)$ (.) for the skew symmetric part of the differential operator. The state space is defined as $X=$ $L_{2}\left(a, b ; \mathbb{R}^{n}\right)$ with inner product $\left\langle x_{1}, x_{2}\right\rangle_{\mathcal{L}}=\left\langle x_{1}, \mathcal{L} x_{2}\right\rangle$ and norm $\left\|x_{1}\right\|_{\mathcal{L}}^{2}=\left\langle x_{1}, x_{1}\right\rangle_{\mathcal{L}}$. Hence $X$ is a Hilbert space. Note that the natural norm on $X$ and the $L_{2}$ norm are equivalent. The reason for selecting this space is that $\|\cdot\|^{2}$ is usually related to the energy function of the system.

Definition II.1. [5], [7] Let $\mathcal{L} x \in H^{1}\left(a, b ; \mathbb{R}^{n}\right)$. Then the boundary port variables associated with system (1) are the vectors $e_{\partial, \mathcal{L} x}, f_{\partial, \mathcal{L} x} \in \mathbb{R}^{n}$, defined by

$$
\left[\begin{array}{l}
f_{\partial, \mathcal{L} x} \\
e_{\partial, \mathcal{L} x}
\end{array}\right]=\frac{1}{\sqrt{2}}\left[\begin{array}{cc}
P_{1} & -P_{1} \\
I & I
\end{array}\right]\left[\begin{array}{l}
(\mathcal{L} x)(b) \\
(\mathcal{L} x)(a)
\end{array}\right]=R\left[\begin{array}{l}
(\mathcal{L} x)(b) \\
(\mathcal{L} x)(a)
\end{array}\right] .
$$

Note that the port variables are linear combinations of the boundary variables. Let us define the matrix $\Sigma \in M_{2 n}(\mathbb{R})$ as follows

$$
\Sigma=\left[\begin{array}{ll}
0 & I \\
I & 0
\end{array}\right] \text {. }
$$

Theorem II.1. [5], [7] Let $W$ be a $n \times 2 n$ real matrix. If $W$ has full rank and satisfies $W \Sigma W^{\top} \geq 0$, where $\Sigma$ is defined in (3), then (1), with input

$$
u(t)=W\left[\begin{array}{l}
f_{\partial, \mathcal{L} x}(t) \\
e_{\partial, \mathcal{L} x}(t)
\end{array}\right]
$$

is a boundary control system on $X$ in the sense of [2, page 122]. Furthermore, the operator $\mathcal{A} x=P_{1}(\partial / \partial z)(\mathcal{L} x)+\left(P_{0}-G_{0}\right) \mathcal{L} x$ with domain

$$
D(\mathcal{A})=\left\{\mathcal{L} x \in H^{1}\left(a, b ; \mathbb{R}^{n}\right) \mid\left[\begin{array}{l}
f_{\partial, \mathcal{L} x} \\
e_{\partial, \mathcal{L} x}
\end{array}\right] \in \operatorname{ker} W\right\}
$$

generates a contraction semigroup on $X$. Let $\tilde{W}$ be a full rank matrix of size $n \times 2 n$ with $\left[\begin{array}{c}W \\ \tilde{W}\end{array}\right]$ invertible and let $P_{W, \tilde{W}}$ be given by

$$
P_{W, \tilde{W}}=\left(\left[\begin{array}{c}
W \\
\tilde{W}
\end{array}\right] \Sigma\left[\begin{array}{c}
W \\
\tilde{W}
\end{array}\right]^{\top}\right)^{-1}=\left[\begin{array}{ll}
W \Sigma W^{\top} & W \Sigma \tilde{W}^{\top} \\
\tilde{W} \Sigma W^{\top} & \tilde{W} \Sigma \tilde{W}^{\top}
\end{array}\right]^{-1} .
$$

Preprint submitted to IEEE Transactions on Automatic Control. Received: April 2, 2014 09:18:44 PST 
Define the output of the system as the linear mapping $\mathcal{C}$ : $\mathcal{L}^{-1} H^{1}\left(a, b ; \mathbb{R}^{n}\right) \rightarrow \mathbb{R}^{n}$,

$$
y(t)=\mathcal{C} x(t):=\tilde{W}\left[\begin{array}{l}
f_{\partial, \mathcal{L} x}(t) \\
e_{\partial, \mathcal{L} x}(t)
\end{array}\right] .
$$

Then for $u \in C^{2}\left(0, \infty ; \mathbb{R}^{k}\right), \mathcal{L} x(0) \in H^{1}\left(a, b ; \mathbb{R}^{n}\right)$, and $u(0)=$ $W\left[\begin{array}{l}f_{\partial, \mathcal{L}_{x}}(0) \\ e_{\partial, \mathcal{L}_{x}}(0)\end{array}\right]$ the following balance equation is satisfied:

$$
\begin{aligned}
\frac{1}{2} \frac{d}{d t}\|x(t)\|_{\mathcal{L}}^{2} & =\frac{1}{2}\left[\begin{array}{l}
u(t) \\
y(t)
\end{array}\right]^{\top} P_{W, \tilde{W}}\left[\begin{array}{l}
u(t) \\
y(t)
\end{array}\right]-\left\langle G_{0} \mathcal{L} x(t), \mathcal{L} x(t)\right\rangle \\
& \leq \frac{1}{2}\left[\begin{array}{l}
u(t) \\
y(t)
\end{array}\right]^{\top} P_{W, \tilde{W}}\left[\begin{array}{l}
u(t) \\
y(t)
\end{array}\right]
\end{aligned}
$$

The matrix $P_{W, \tilde{W}}$ is defined if and only if $\left[\begin{array}{c}W \\ \tilde{W}\end{array}\right]$ is invertible. Notice that in the absence of some internal dissipation $\left(G_{0}=0\right)$ the system only exchanges energy with the environment through the boundaries since the input and output act on the boundary of the spatial domain. Finally, we remark that the balance equation (4) represents the energy balance equation relating the internal energy variation to the power flow at the boundary and may be rewritten as

$$
\frac{1}{2} \frac{d}{d t}\|x(t)\|_{\mathcal{L}}^{2} \leq \frac{1}{2}\left[\begin{array}{l}
(\mathcal{L} x)(t, b) \\
(\mathcal{L} x)(t, a)
\end{array}\right]^{\top}\left[\begin{array}{cc}
P_{1} & 0 \\
0 & -P_{1}
\end{array}\right]\left[\begin{array}{l}
(\mathcal{L} x)(t, b) \\
(\mathcal{L} x)(t, a)
\end{array}\right]
$$

with $\left[\begin{array}{cc}P_{1} & 0 \\ 0 & -P_{1}\end{array}\right]=R^{\top} \Sigma R$.

Remark II.1. As it has been pointed out in [8, page 36-41], if the matrices $W$ and $\tilde{W}$ are selected such that $P_{W, \tilde{W}}=\left[\begin{array}{ll}0 & I \\ I & 0\end{array}\right]=\Sigma$, then the BCS fulfils $\frac{1}{2} \frac{d}{d t}\|x(t)\|_{\mathcal{L}}^{2} \leq u^{\top}(t) y(t)$, and is called an impedance energy preserving $B C S$.

We follow [6], [8] to define a class of BCS on some extended state space $\tilde{X}=X \times V$, where $V$ is the state space of a finite dimensional control system interconnected with the BCS of Theorem II.1 through its boundaries. Consider a linear system with minimal realization

$$
\dot{v}=A_{c} v+B_{c} u_{c}, \quad y_{c}=C_{c} v+D_{c} u_{c},
$$

with state $v \in V=\mathbb{R}^{m}$, input values $u_{c} \in U_{c}=\mathbb{R}^{n}$ and output values $y_{c} \in Y_{c}=\mathbb{R}^{n}$. Moreover, $A_{c}, B_{c}, C_{c}$ and $D_{c}$ are constant real matrices of dimension $m \times m, m \times n, n \times m$ and $n \times n$ respectively.

Assumption 1. The linear control system is strictly positive real (SPR) [11], [12], i.e. $\sigma_{\min }\left(B_{c}\right)>0$ and there exist matrices $P_{c}>0$, $P_{c}, L \in \mathbb{R}^{m \times m}, \mu_{\text {min }}(L) \triangleq \epsilon>0, M_{c} \in \mathbb{R}^{n \times m}, N_{c} \in \mathbb{R}^{m \times m}$ that satisfy the Lur'e equations:

$$
\begin{aligned}
A_{c}^{\top} P_{c}+P_{c} A_{c} & =-M_{c}^{\top} M_{c}-L_{c}, \quad B_{c}^{\top} P_{c}-C_{c}=N_{c}^{\top} M_{c}, \\
N_{c}^{\top} N_{c} & =D_{c}+D_{c}^{\top}
\end{aligned}
$$

The existence of a solution to (7) implies that (6) fulfils the so called dissipation inequality [12], [13] $E_{c}(v(t)) \leq E_{c}(v(0))+$ $\int_{0}^{t} w(u(s), y(s)) d s$, where $E_{c}(v) \geq 0$ is called the storage function (for physical systems it usually corresponds to the energy), and $w(u, y)$ is called the supply rate. If the supply rate is of the form $w(u, y)=u^{\top} y-u^{\top} S u$, with $S=\sigma I, \sigma>0$, then the dissipative system is called input-strictly passive. In [6], [8] it is shown that a power conserving interconnection [14] (Fig. 1), i.e.,

$$
u=r-y_{c}, \quad y=u_{c},
$$

with $r \in \mathbb{R}^{n}$ the new input of the system, of an impedance energy preserving BCS and a linear SPR finite dimensional system defines again a BCS on the extended space $\tilde{X}$.

Theorem II.2. [6], [8] Let the state of the open-loop BCS satisfy $\frac{1}{2} \frac{d}{d t}\|x(t)\|_{\mathcal{L}}^{2} \leq u^{\top}(t) y(t)$. Consider a LTI SPR finite dimensional system with storage function $E_{c}(t)=\frac{1}{2}\left\langle v(t), Q_{c} v(t)\right\rangle_{\mathbb{R}^{m}}, Q_{c}=$

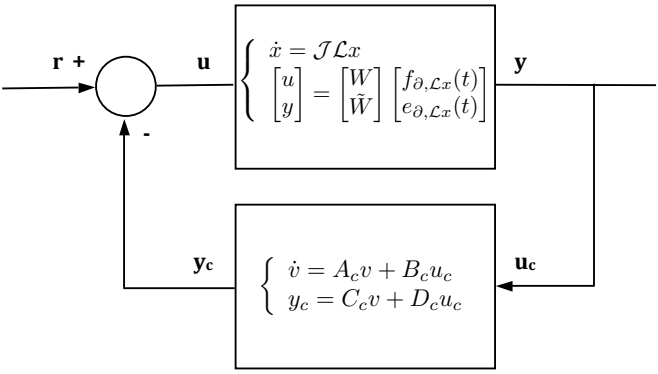

Fig. 1. Power preserving interconnection

$Q_{c}^{\top}>0 \in M_{m}(\mathbb{R})$. Then the feedback interconnection of the BCS and the finite dimensional system is again a BCS on the extended state space $\tilde{x} \in \tilde{X}=X \times V$ with inner product $\left\langle\tilde{x}_{1}, \tilde{x}_{2}\right\rangle_{\tilde{X}}=$ $\left\langle x_{1}, x_{2}\right\rangle_{\mathcal{L}}+\left\langle v_{1}, Q_{c} v_{2}\right\rangle_{V}$. Furthermore, the operator $\mathcal{A}_{e}$ defined by

$$
\mathcal{A}_{e} \tilde{x}=\left[\begin{array}{cc}
\mathcal{J} \mathcal{L} & 0 \\
B_{c} \mathcal{C} & A_{c}
\end{array}\right]\left[\begin{array}{l}
x \\
v
\end{array}\right]
$$

with

$D\left(\mathcal{A}_{e}\right)=\left\{\left[\begin{array}{l}x \\ v\end{array}\right] \in\left[\begin{array}{l}X \\ V\end{array}\right] \mid \mathcal{L} x \in H^{N}\left(a, b ; \mathbb{R}^{n}\right),\left[\begin{array}{c}f_{\partial, \mathcal{L} x} \\ e_{\partial, \mathcal{L} x} \\ v\end{array}\right] \in\right.$ ker $\left.\tilde{W}_{D}\right\}$,

where $\tilde{W}_{D}=\left[\left(\begin{array}{ll}W+D_{c} \tilde{W} & C_{c}\end{array}\right)\right]$ generates a contraction semigroup on $\tilde{X}$.

\section{DYNAMIC BOUNDARY CONTROL}

The main result of this paper is the use of strictly-input passive finite dimensional boundary controllers to exponentially stabilize a large set of BCS. This is a powerful result due to two reasons: 1) Exponential stability is a very strong condition that in general is difficult to prove, but that provides a measure of performance associated with the time decay constant. 2) Many physical applications are modelled as infinite dimensional systems coupled at the boundaries with finite dimensional systems, i.e., set of coupled PDE's and ODE's. In those cases the finite dimensional part (ODE) corresponds to the "controller" and the stability of the coupled system may be analysed using our approach. We will focus our study in a particular class of linear boundary controllers, namely port-Hamiltonian control systems, since a large class of physical applications are naturally modelled using the port-Hamiltonian formalism [14].

Proposition III.1. A finite dimensional control system

$$
\dot{v}=\left(J_{c}-R_{c}\right) Q_{c} v+B_{c} u_{c}, \quad y_{c}=B_{c}^{\top} Q_{c} v+S_{c} u_{c},
$$

is exponentially stable and satisfies Assumption 1, i.e., is SPR, if there exist constant matrices of proper dimension $Q_{c}=Q_{c}^{\top}>0$, $J_{c}=-J_{c}^{\top}, R_{c}=R_{c}^{\top} \geq 0, S_{c}=S_{c}^{\top}>0, B_{c}$ full rank and $A_{c}:=\left(J_{c}-R_{c}\right) Q_{c}$ Hurwitz. Consequently, system (9) is a strictly input passive port-Hamiltonian system, i.e., there exists $\sigma>0$ such that $\dot{E}_{c}(t) \leq u_{c}(t)^{\top} y_{c}(t)-\sigma\left\|u_{c}(t)\right\|^{2}$.

We shall frequently use the following inequalities for $v, w \in \mathbb{R}^{n}$ and $\alpha>0$

$$
\begin{aligned}
-\alpha^{2}\|v\|^{2}-\frac{1}{\alpha^{2}}\|w\|^{2} & \leq v^{\top} w+w^{\top} v \\
& \leq \alpha^{2}\|v\|^{2}+\frac{1}{\alpha^{2}}\|w\|^{2} .
\end{aligned}
$$

This holds since $\left\|\alpha v \pm \frac{1}{\alpha} w\right\|^{2} \geq 0$. The following Lemmas follow from Proposition III.1.

\footnotetext{
${ }^{1}$ This is equivalent to the pair $\left(J_{c}, R_{c}\right)$ being controllable
} 
Lemma III.2. There exist strictly positive constants $\kappa_{2}, \kappa_{4}$ and $\kappa_{5}$ such that for all $\tau>0$ the energy of (9) satisfies:

$$
E_{c}(\tau) \leq \kappa_{1}(\tau) E_{c}(0)+\kappa_{5} \int_{0}^{\tau}\left\|u_{c}(t)\right\|^{2} d t
$$

where $\kappa_{1}(\tau)=\kappa_{4} e^{-\kappa_{2} \tau}$.

Proof: Since $A_{c}=\left(J_{c}-R_{c}\right) Q_{c}$ is exponentially stable there exists $\kappa_{2}>0$ such that $A_{c}+2 \kappa_{2} I$ is still exponentially stable. Hence there exists a $P_{1}=P_{1}^{\top}>0$ satisfying

$$
\left(A_{c}+2 \kappa_{2} I\right)^{\top} P_{1}+P_{1}\left(A+2 \kappa_{2} I\right) \leq 0
$$

which implies that $A_{c}^{\top} P_{1}+P_{1} A_{c} \leq-4 \kappa_{2} P_{1}$. Taking the time derivative of $v^{\top} P_{1} v$ along trajectories we have

$$
\begin{aligned}
\frac{d}{d t}\left(v^{\top} P_{1} v\right) & =\left(A_{c} v+B_{c} u_{c}\right)^{\top} P_{1} v+v^{\top} P_{1}\left(A_{c} v+B_{c} u_{c}\right) \\
& \leq-4 \kappa_{2} v^{\top} P_{1} v+u_{c}^{\top} B_{c}^{\top} P_{1} v+v^{\top} P_{1} B_{c} u_{c} \\
& \leq-2 \kappa_{2} v^{\top} P_{1} v+\kappa_{3}\left\|u_{c}\right\|^{2}
\end{aligned}
$$

for some $\kappa_{3}>0$, where we used (11) with $\alpha^{2}=2 \kappa_{2}$. This implies that

$$
\frac{d}{d t}\left(e^{\kappa_{2} t} v^{\top}(t) P_{1} v(t)\right) \leq \kappa_{3} e^{\kappa_{2} t}\left\|u_{c}(t)\right\|^{2} .
$$

Integrating this relation over $t \in[0, \tau]$ and rearranging terms, we obtain $v(\tau)^{\top} P_{1} v(\tau) \leq e^{-\kappa_{2} \tau} v(0)^{\top} P_{1} v(0)+$ $\int_{0}^{\tau} \kappa_{3} e^{\kappa_{2}(t-\tau)}\left\|u_{c}(t)\right\|^{2} d t$. Using Lemma A.6.6 from [2, page 638] we have that $\int_{0}^{\tau} \kappa_{3} e^{\kappa_{2}(t-\tau)}\left\|u_{c}(t)\right\|^{2} d t \leq \kappa_{5} \int_{0}^{\tau}\left\|u_{c}(t)\right\|^{2} d t$ for some $\kappa_{5}>0$, and since there exists positive constants $q_{1}, q_{2}$ such that for all $v \in \mathbb{R}^{n} q_{1} v^{\top} P_{1} v \leq \frac{1}{2} v^{\top} Q_{c} v \leq q_{2} v^{\top} P_{1} v$, inequality (12) follows.

Lemma III.3. There exists positive constants $\xi_{1}, \xi_{2}$ and $\tau_{0}$ such for all $\tau>\tau_{0}$ the energy of (9) satisfies

$$
\int_{0}^{\tau} E_{c}(t) d t \leq \xi_{1} \int_{0}^{\tau} v^{\top}(t) Q_{c} R_{c} Q_{c} v(t) d t+\xi_{2} \int_{0}^{\tau}\left\|u_{c}(t)\right\|^{2} d t
$$

Proof: Since $A_{c}=\left(J_{c}-R_{c}\right) Q_{c}$ is exponentially stable there exists a $P_{2}=P_{2}^{\top} \geq 0$ such that $A_{c}^{\top} P_{2}+P_{2} A_{c}=-\frac{1}{2} Q_{c}$. Taking the time derivative of $v^{\top} P_{2} v$ we have

$$
\begin{aligned}
\frac{d}{d t}\left(v^{\top} P_{2} v\right) & =\left(A_{c} v+B_{c} u_{c}\right)^{\top} P_{2} v+v^{\top} P_{2}\left(A_{c} v+B_{c} u_{c}\right) \\
& =-\frac{1}{2} v^{\top} Q_{c} v+u_{c}^{\top} B_{c}^{\top} P_{2} v+v^{\top} P_{2} B_{c} u_{c} .
\end{aligned}
$$

Now, using equation (11) we find

$$
\frac{d}{d t}\left(v^{\top} P_{2} v\right) \leq\left(\alpha^{2}-1\right) \frac{1}{2} v^{\top} Q_{c} v+\frac{\beta_{3}}{\alpha^{2}} u_{c}^{\top} u_{c}
$$

for some $\beta_{3}>0$ and $\left.\alpha \in\right] 0,1$ [. Now, integrating (17) we obtain

$$
\begin{aligned}
& \left(v^{\top} P_{2} v\right)(\tau)-\left(v^{\top} P_{2} v\right)(0) \leq \\
& \left(\alpha^{2}-1\right) \int_{0}^{\tau} E_{c}(t) d t+\frac{\beta_{3}}{\alpha^{2}} \int_{0}^{\tau}\left\|u_{c}(t)\right\|^{2} d t .
\end{aligned}
$$

Since $P_{2}$ and $Q_{c}$ are symmetric and positive we may bound them by $P_{2} \leq \frac{1}{2} \beta_{5} Q_{c}$, with $\beta_{5}>0$ sufficiently large, to obtain

$$
\int_{0}^{\tau} E_{c}(t) d t \leq \beta_{1} E_{c}(0)+\beta_{2} \int_{0}^{\tau}\left\|u_{c}(t)\right\|^{2} d t .
$$

with $\beta_{1}=\frac{\beta_{5}}{\left(1-\alpha^{2}\right)}>0$ and $\beta_{2}=\frac{1}{\left(1-\alpha^{2}\right)} \frac{\beta_{3}}{\alpha^{2}}>0$. On the other hand we have that the time derivative of the energy of (9) is given by

$$
\begin{aligned}
& \frac{1}{2} \frac{d}{d t}\left(v^{\top} Q_{c} v\right)= \\
& \quad-v^{\top} Q_{c} R_{c} Q_{c} v+\frac{1}{2}\left(u_{c}^{\top} B_{c}^{\top} Q_{c} v+v^{\top} Q_{c} B_{c} u_{c}\right) .
\end{aligned}
$$

Using (10) in (20) we obtain we obtain for any $\eta>0$

$$
\begin{aligned}
& \frac{1}{2} \frac{d}{d t}\left(v^{\top} Q_{c} v\right) \geq \\
& \quad-v^{\top} Q_{c} R_{c} Q_{c} v-\frac{1}{2}\left(\eta^{2}\left\|Q_{c}^{\frac{1}{2}} B_{c} u_{c}\right\|^{2}+\frac{1}{\eta^{2}}\left\|Q_{c}^{\frac{1}{2}} v\right\|^{2}\right)
\end{aligned}
$$

Integrating and grouping terms

$$
\begin{aligned}
& E_{c}(0) \leq E_{c}(\tau)+ \\
& \quad \int_{0}^{\tau} v(t)^{\top} Q_{c} R_{c} Q_{c} v(t)+\frac{\eta^{2}}{2}\left\|Q_{c}^{\frac{1}{2}} B_{c} u_{c}(t)\right\|^{2}+\frac{1}{2 \eta^{2}} E_{c}(t) d t .
\end{aligned}
$$

Now, applying Lemma III.2 and using (19) we obtain

$$
\begin{aligned}
E_{c}(0) \leq & \kappa_{1}(\tau) E_{c}(0)+\kappa_{3} \int_{0}^{\tau}\left\|u_{c}(t)\right\|^{2} d t \\
& +\int_{0}^{\tau} v^{\top} Q_{c} R_{c} Q_{c} v+\frac{\eta^{2}}{2}\left\|Q_{c}^{\frac{1}{2}} B_{c}\right\|^{2}\left\|u_{c}(t)\right\|^{2} d t \\
& \quad+\frac{1}{2 \eta^{2}}\left(\beta_{1} E_{c}(0)+\beta_{2} \int_{0}^{\tau}\left\|u_{c}(t)\right\|^{2} d t\right) .
\end{aligned}
$$

Choosing $\tau_{0}$ and $\eta$ sufficiently large, such that $\kappa_{1}\left(\tau_{0}\right)+\frac{\beta_{1}}{2 \eta^{2}}<1$, see Lemma III.2, we obtain for $\tau>\tau_{0}$

$$
E_{c}(0) \leq \gamma_{1} \int_{0}^{\tau} v(t)^{\top} Q_{c} R_{c} Q_{c} v(t) d t+\gamma_{2} \int_{0}^{\tau}\left\|u_{c}(t)\right\|^{2} d t,
$$

with constants $\gamma_{1}=1 /\left(1-\left(\kappa_{1}\left(\tau_{0}\right)+\frac{\beta_{1}}{2 \eta^{2}}\right)\right)$ and $\gamma_{2}=$ $\left(\kappa_{3}+\frac{\eta^{2}}{2}\left\|Q_{c}^{\frac{1}{2}} B_{c}\right\|^{2}+\frac{\beta_{2}}{2 \eta^{2}}\right) \gamma_{1}$. Now, combining (19) and (21) we obtain

$$
\int_{0}^{\tau} E_{c}(t) d t \leq \xi_{1} \int_{0}^{\tau} v^{\top} Q_{c} R_{c} Q_{c} v d t+\xi_{2} \int_{0}^{\tau}\left\|u_{c}(t)\right\|^{2} d t
$$

with $\xi_{1}=\beta_{1} \gamma_{1}$ and $\xi_{2}=\beta_{1} \gamma_{2}+\beta_{2}$, which proves the Lemma.

Lemma III.4. For every $\delta_{1}>0$ there exists a $\delta_{2}>0$ such that for all $\tau>0$ the energy of (9) satisfies the relation

$$
\int_{0}^{\tau} \delta_{1} E_{c}(t)+\left\|y_{c}(t)\right\|^{2} d t \leq \delta_{2} \int_{0}^{\tau} E_{c}(t)+\left\|u_{c}(t)\right\|^{2} d t .
$$

Proof: The relation follows by noting that the left term of (23) may be written and bounded as

$$
\begin{array}{r}
\int_{0}^{\tau}\left[\begin{array}{c}
v \\
u_{c}
\end{array}\right]^{\top}\left[\begin{array}{rr}
\left(\frac{\delta_{1}}{2} Q_{c}+Q_{c} B_{c} B_{c}^{\top} Q_{c}\right) & Q_{c} B_{c} S_{c} \\
S_{c} B_{c}^{\top} Q_{c} & S_{c}^{2}
\end{array}\right]\left[\begin{array}{c}
v \\
u_{c}
\end{array}\right] d t \\
\leq \delta_{2} \int_{0}^{\tau}\left[\begin{array}{c}
v \\
u_{c}
\end{array}\right]^{\top}\left[\begin{array}{cc}
\frac{1}{2} Q_{c} & 0 \\
0 & I
\end{array}\right]\left[\begin{array}{c}
v \\
u_{c}
\end{array}\right] d t
\end{array}
$$

for some $\delta_{2}>0$.

\section{EXPONENTIAL STABILITY}

To show that the BCS defined in Theorem II.2 is exponentially stable, we first show that the total energy of the coupled PDE-ODE system is bounded by the energy flowing through the boundaries of the infinite dimensional part and the stored energy in the finite dimensional part. Then using this bound it is shown that the total energy decays exponentially. In the rest of the paper, we will set $G_{0}=$ 0 for simplicity. Notice, however, that all results are valid for $G_{0} \neq 0$. Moreover, as in the case of finite dimensional systems, the presence of natural dissipation adds in general robustness to the control. As part of the boundary port variables of the infinite dimensional system can be set to zero (and hence not used for the interconnection) we will assume that the infinite dimensional system satisfies a dissipative relation. 
Assumption 2. The BCS of Theorem II.1 satisfies $\|u(t)\|^{2}+$ $\|y(t)\|^{2} \geq \epsilon\|\mathcal{L} x(t, b)\|^{2} \quad\left(\right.$ or $\left.\geq \epsilon\|\mathcal{L} x(t, a)\|^{2}\right)$ for some $\epsilon>0$.

The physical interpretation of Assumption 2 is that the energy flowing through any of the boundaries is bounded by the energy flowing in/out through the inputs/outputs. This is, roughly speaking, related to the passivity of (1) and to the definition of inputs and outputs given in Theorem II.1. The following Lemma gives a bound on the total energy of the interconnected system.

Lemma IV.1. Consider a BCS as described in Theorem II.2 with $r(t)=0$, for all $t \geq 0$. Then, the energy of the system $\tilde{E}(t)=$ $\frac{1}{2}\|x(t)\|_{\mathcal{L}}^{2}+\frac{1}{2} v(t)^{T} Q_{c} v(t)$ satisfies for $\tau$ large enough

$$
\begin{aligned}
& \tilde{E}(\tau) \leq c(\tau) \int_{0}^{\tau}\|(\mathcal{L} x)(t, b)\|^{2} d t+\frac{2 c(\tau)}{c_{1}} \int_{0}^{\tau} E_{c}(t) d t, \\
& \tilde{E}(\tau) \leq c(\tau) \int_{0}^{\tau}\|(\mathcal{L} x)(t, a)\|^{2} d t+\frac{2 c(\tau)}{c_{1}} \int_{0}^{\tau} E_{c}(t) d t,
\end{aligned}
$$

where $c$ is a positive constant that only depends on $\tau$ and $c_{1}$ is a positive constant.

Proof: In [7], it has been proved that there exist positive $\gamma$ and $\tau_{1}$ such that for $\tau>\tau_{1}>2 \gamma(b-a)$, the function $F(z)=$ $\int_{\gamma(b-z)}^{\tau-\gamma(b-z)} x^{\top}(t, z) \mathcal{L}(z) x(t, z) d t$ fulfils $F(b) \geq F(z) e^{-\kappa(b-a)}$ for $z \in[a, b]$ where $\kappa$ is a positive constant. On other hand, due to the contraction property of the semigroup $\tilde{E}\left(t_{2}\right) \leq \tilde{E}\left(t_{1}\right)$ for $t_{2} \geq t_{1}$ it is deduced that

$$
\begin{aligned}
\int_{\gamma(b-a)}^{\tau-\gamma(b-a)} \tilde{E}(t) d t & \geq \tilde{E}(\tau-\gamma(b-a)) \int_{\gamma(b-a)}^{\tau-\gamma(b-a)} d t \\
& \geq(\tau-2 \gamma(b-a)) \tilde{E}(\tau-\gamma(b-a)) .
\end{aligned}
$$

Hence we obtain

$$
\begin{aligned}
2(\tau & -2 \gamma(b-a)) \tilde{E}(\tau) \\
& \leq 2(\tau-2 \gamma(b-a)) \tilde{E}(\tau-\gamma(b-a)) \\
& \leq \int_{a}^{b}\left(\int_{\gamma(b-z)}^{\tau-\gamma(b-z)} x^{\top}(\mathcal{L} x) d t\right) d z+2 \int_{\gamma(b-z)}^{\tau-\gamma(b-z)} E_{c} d t \\
& \leq \int_{a}^{b} F(z) d z+2 \int_{\gamma(b-z)}^{\tau-\gamma(b-z)} E_{c} d t \\
& \leq(b-a) F(b) e^{\kappa(b-a)}+2 \int_{\gamma(b-z)}^{\tau-\gamma(b-z)} E_{c} d t \\
& \leq c_{1} \int_{0}^{\tau}\|(\mathcal{L} x)(b)\|^{2} d t+2 \int_{\gamma(b-z)}^{\tau-\gamma(b-z)} E_{c} d t
\end{aligned}
$$

where $c_{1}=(b-a)\left\|\mathcal{L}^{-1}(b)\right\| e^{\kappa(b-a)}$, and where Fubini's theorem and the fact that the integration interval increases when substituting $a$ by $z$ in the integral of the energy have been used in the second step. Hence we obtain that for $\tau>\tau_{1}, \tilde{E}(\tau) \leq c(\tau) \int_{0}^{\tau}\|(\mathcal{L} x)(t, b)\|^{2} d t+$ $\frac{2 c(\tau)}{c_{1}} \int_{\gamma(b-a)}^{\tau-\gamma(b-a)} E_{c}(t) d t$, with $c(\tau)=\frac{c_{1}}{2(\tau-2 \gamma(b-a))}$. The second inequality is obtained similarly with $F(z)=\int_{\gamma(a-z)}^{\tau-\gamma(a-z)} x^{\top} \mathcal{L} x d t$.

Theorem IV.2. Consider the BCS defined by Theorem II.2 with $r(t)=0$, for all $t \geq 0$. The finite dimensional boundary controller of Proposition III.1 exponentially stabilizes the BCS.

Proof: Let $\sigma>0$ be such that $S_{c} \geq \sigma I$. The time derivative of the total energy satisfies

$$
\begin{aligned}
\dot{\tilde{E}} & =-v^{\top} Q_{c} R_{c} Q_{c} v-u_{c}^{\top} S_{c} u_{c} \\
& \leq-v^{\top} Q_{c} R_{c} Q_{c} v-\sigma u_{c}^{\top} u_{c}, \quad \text { since } S_{c} \geq \sigma I \\
& =-v^{\top} Q_{c} R_{c} Q_{c} v-\sigma \epsilon_{1} u_{c}^{\top} u_{c}-\sigma \epsilon_{2} u_{c}^{\top} u_{c} \\
& =-v^{\top} Q_{c} R_{c} Q_{c} v-\sigma \epsilon_{1}\left\|u_{c}\right\|^{2}-\sigma \epsilon_{2}\|y\|^{2} \\
& =-v^{\top} Q_{c} R_{c} Q_{c} v-\sigma \epsilon_{1}\left\|u_{c}\right\|^{2}-\sigma \epsilon_{2}\left(\|y\|^{2}+\|u\|^{2}\right)+\sigma \epsilon_{2}\|u\|^{2}
\end{aligned}
$$

with $\epsilon_{1}+\epsilon_{2}=1$ and where we have used that $u_{c}=-y$. Using Assumption 2 we have

$$
\dot{\tilde{E}} \leq-v^{\top} Q_{c} R_{c} Q_{c} v-\sigma \epsilon_{1}\left\|u_{c}\right\|^{2}-\sigma \epsilon_{2} \epsilon\|\mathcal{L} x(t, b)\|^{2}+\sigma \epsilon_{2}\left\|y_{c}\right\|^{2} .
$$

Integrating this equation on $t \in[0, \tau]$ we have

$$
\begin{aligned}
& \tilde{E}(\tau)-\tilde{E}(0) \leq-\int_{0}^{\tau} v^{\top}(t) Q_{c} R_{c} Q_{c} v(t) d t \\
& +\int_{0}^{\tau}-\sigma \epsilon_{1}\left\|u_{c}(t)\right\|^{2}-\sigma \epsilon_{2} \epsilon\|\mathcal{L} x(t, b)\|^{2}+\sigma \epsilon_{2}\left\|y_{c}(t)\right\|^{2} d t .
\end{aligned}
$$

Next choose $\tau$ sufficiently large such that Lemmas III.3 and IV.1 hold. Using the latter lemma we have

$$
\begin{aligned}
& \tilde{E}(\tau)-\tilde{E}(0) \leq-\int_{0}^{\tau} v^{\top} Q_{c} R_{c} Q_{c} v+\sigma \epsilon_{1}\left\|u_{c}\right\|^{2} d t \\
& \quad+\frac{\sigma \epsilon_{2} \epsilon}{c(\tau)}\left(\frac{2 c(\tau)}{c_{1}} \int_{0}^{\tau} E_{c}(t) d t-\tilde{E}(\tau)\right)+\sigma \epsilon_{2} \int_{0}^{\tau}\left\|y_{c}\right\|^{2} d t .
\end{aligned}
$$

Grouping terms we have that

$$
\begin{aligned}
& \tilde{E}(\tau)\left(1+\frac{\sigma \epsilon_{2} \epsilon}{c(\tau)}\right)-\tilde{E}(0) \leq \\
& \quad-\int_{0}^{\tau} v(t)^{\top} Q_{c} R_{c} Q_{c} v(t) d t-\sigma \epsilon_{1} \int_{0}^{\tau}\left\|u_{c}(t)\right\|^{2} d t \\
& \quad+\sigma \epsilon_{2}\left(\int_{0}^{\tau} \frac{2 \epsilon}{c_{1}} E_{c}(t)+\left\|y_{c}(t)\right\|^{2} d t\right) .
\end{aligned}
$$

Using Lemma III.4 with $\delta_{1}=\frac{2 \epsilon}{c_{1}}$ we have

$$
\begin{array}{r}
\tilde{E}(\tau)\left(1+\frac{\sigma \epsilon_{2} \epsilon}{c(\tau)}\right)-\tilde{E}(0) \leq-\int_{0}^{\tau} v(t)^{\top} Q_{c} R_{c} Q_{c} v(t) d t \\
+\sigma \epsilon_{2} \delta_{2} \int_{0}^{\tau} E_{c}(t) d t+\sigma\left(\epsilon_{2} \delta_{2}-\epsilon_{1}\right) \int_{0}^{\tau}\left\|u_{c}(t)\right\|^{2} d t .
\end{array}
$$

Now, using Lemma III.3 we obtain

$$
\begin{aligned}
& \tilde{E}(\tau)\left(1+\frac{\sigma \epsilon_{2} \epsilon}{c(\tau)}\right)-\tilde{E}(0) \leq \\
& \left(\sigma \epsilon_{2} \delta_{2} \xi_{1}-1\right) \int_{0}^{\tau} v(t)^{\top} Q_{c} R_{c} Q_{c} v(t) d t \\
& +\sigma\left(\epsilon_{2} \delta_{2}\left(1+\xi_{2}\right)-\epsilon_{1}\right) \int_{0}^{\tau}\left\|u_{c}(t)\right\|^{2} d t
\end{aligned}
$$

Since $\epsilon_{2}$ may be chosen to be arbitrarily small, i.e, $\epsilon_{2} \ll 1$ and since $\epsilon_{1}=1-\epsilon_{2}$, we have that $\tilde{E}(\tau) \leq c_{2} \tilde{E}(0)$ with $c_{2}=\frac{1}{\left(1+\frac{\sigma \epsilon_{2} \epsilon}{c(\tau)}\right)}<1$. From this we see that the semigroup $T(t)$ generated by $\mathcal{A}_{e}$ satisfies $\|T(\tau)\| \leq 1$ for a sufficiently large $\tau$. Consequently, $\log (\|T(\tau)\|) \leq$ 0 , and by using the properties of strongly continuous semigroups [2, Theorem 2.16, page 18] there exist $\tilde{M}$ and $\alpha>0$ such that $\|T(t)\| \leq \tilde{M} e^{-\alpha t}$ for all $t>0$.

\section{EXAMPLE: DNA-MANIPULATION PROCESS}

In this section we illustrate the exponential stability result on a physically based example: a controlled nanotweezer used for DNA manipulation [15]. The considered class of tweezer is presented in Figure 2, and the control objective is to control the translational position of the DNA-bundle $\left(x_{c 2}\right)$. From physical considerations the lumped model of the DNA bundle is split into two parts: a longitudinal model and a rotation model. The longitudinal model accounts for mean viscous frictions of the wet molecules and for the longitudinal motion (with inertia) of the bundle. It is composed of a damper interconnected to a moving load attached to a springdamper system. The rotation model accounts for the bending of the DNA sample and is considered with the same structure. The arm is modelled as a Timoshenko beam and is actuated applying a force 


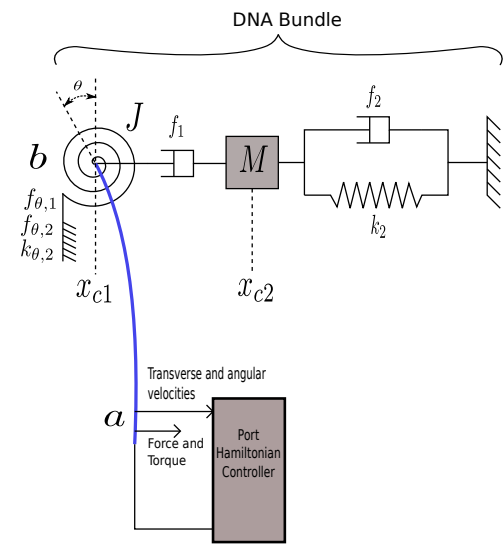

Fig. 2. DNA manipulation with PH control

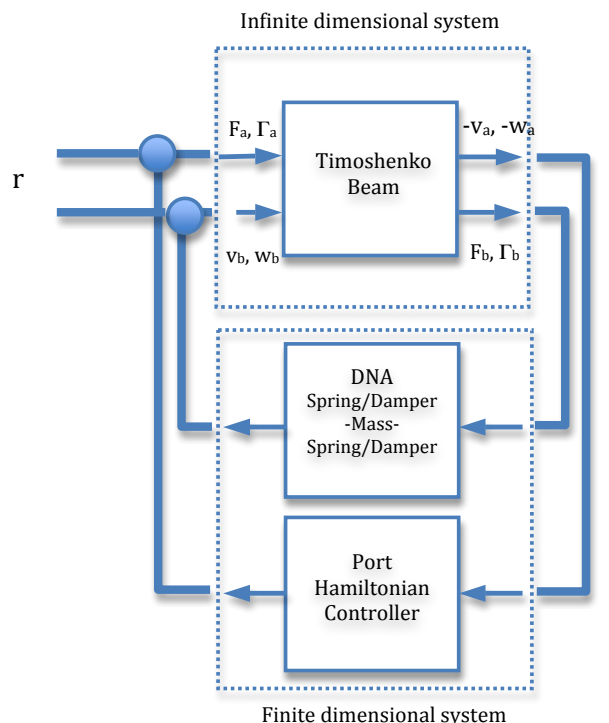

Fig. 3. Closed-loop system

and a torque at point $a$ by using a port-Hamiltonian controller. We assume that it is only possible to measure the transversal and angular velocities at the point $a$. The total system, may be divided into three subsystems: the flexible arm, the DNA-bundle at the tip of the gripper and the port-Hamiltonian controller. The flexible arm model is infinite dimensional while the DNA-bundle and port-Hamiltonian controller are finite dimensional. The subsystems are interconnected through their boundary port variables. The interconnection boundary port variables and the causality (depicted with arrows) are given in Figure 3. It is important to remark that only a part of the finite dimensional system is free to design, namely the port-Hamiltonian controller. The DNA-bundle model is given by the physical process. Hence, in this application the control system is given in part by the physically imposed dynamic boundary conditions.

\section{A. The Timoshenko beam}

The Timoshenko beam has been widely studied as portHamiltonian system [16] and as BCS [5]. The exponential stability of the system has been proved for static boundary feedback in [7],
[8]. The port Hamiltonian model of the beam is given by

$$
\frac{\partial}{\partial t}\left[\begin{array}{l}
x_{1} \\
x_{2} \\
x_{3} \\
x_{4}
\end{array}\right]=\underbrace{\left(\begin{array}{llll}
0 & 1 & 0 & 0 \\
1 & 0 & 0 & 0 \\
0 & 0 & 0 & 1 \\
0 & 0 & 1 & 0
\end{array}\right]}_{P_{1}} \frac{\partial}{\partial z}+\underbrace{\left[\begin{array}{cccc}
0 & 0 & 0 & -1 \\
0 & 0 & 0 & 0 \\
0 & 0 & 0 & 0 \\
1 & 0 & 0 & 0
\end{array}\right]}_{P_{0}})\left[\begin{array}{c}
K x_{1} \\
\frac{1}{\rho} x_{2} \\
E I x_{3} \\
\frac{1}{I_{\rho}} x_{4}
\end{array}\right]
$$

with the following state (energy) variables: $x_{1}=\frac{\partial w}{\partial z}(z, t)-\phi(z, t)$ the shear displacement, $x_{2}=\rho(z) \frac{\partial w}{\partial t}(z, t)$ the transverse momentum distribution, $x_{3}=\frac{\partial \phi}{\partial z}(z, t)$ the angular displacement, and $x_{4}=I_{\rho} \frac{\partial \phi}{\partial t}(z, t)$ the angular momentum distribution, for $z \in(a, b)$, $t \geq 0$, where $w(t, z)$ is the transverse displacement of the beam and $\phi(t, z)$ is the rotation angle of a filament of the beam. The coefficients $\rho(z), I_{\rho}(z), E(z), I(z)$ and $K(z)$ are the mass per unit length, the rotary moment of inertia of a cross section, Young's modulus of elasticity, the moment of inertia of a cross section, and the shear modulus respectively. The matrices $P_{1}$ and $P_{0}$ define the skew-symmetric differential operator of order 1 acting on the state space $X=L_{2}\left(a, b, \mathbb{R}^{4}\right), \mathcal{J}=P_{1} \frac{\partial}{\partial z}+P_{0}$. The energy of the beam is expressed in terms of the energy variables, $E=$ $\frac{1}{2} \int_{a}^{b}\left(K x_{1}^{2}+\frac{1}{\rho} x_{2}^{2}+E I x_{3}^{2}+\frac{1}{I_{\rho}} x_{4}^{2}\right) d z=\frac{1}{2}\|x\|_{\mathcal{L}}^{2}$. The boundary port variables are given accordingly to Definition II.1, (see also [5], [8]),

$$
\left[\begin{array}{l}
f_{\partial, \mathcal{L} x} \\
e_{\partial, \mathcal{L} x}
\end{array}\right]=\left[\begin{array}{c}
\left(\rho^{-1} x_{2}\right)(b)-\left(\rho^{-1} x_{2}\right)(a) \\
\left(K x_{1}\right)(b)-\left(K x_{1}\right)(a) \\
\left(I_{\rho}^{-1} x_{4}\right)(b)-\left(I_{\rho}^{-1} x_{4}\right)(a) \\
\left(E I x_{3}\right)(b)-\left(E I x_{3}\right)(a) \\
\left(K x_{1}\right)(b)+\left(K x_{1}\right)(a) \\
\left(\rho^{-1} x_{2}\right)(b)+\left(\rho^{-1} x_{2}\right)(a) \\
\left(E I x_{3}\right)(b)+\left(E I x_{3}\right)(a) \\
\left(I_{\rho}^{-1} x_{4}\right)(b)+\left(I_{\rho}^{-1} x_{4}\right)(a)
\end{array}\right] .
$$

The physical control ports are given by the force/torque acting at the base of the beam (input), and the translational/angular velocities at the base of the beam (output). All physical input and output ports are hence located on the point $a$ of the beam a. The input and output variables of the flexible arm must coincide with the physical ones, hence we define the following input and outputs $u=\left[\begin{array}{llll}v(b) & \omega(b) & F(a) & T(a)\end{array}\right]^{T}, y=$ $\left[\begin{array}{llll}F(b) & T(b) & -v(a) & -\omega(a)\end{array}\right]^{T}$, which is achieved by defining $u=W\left[\begin{array}{l}f_{\partial, \mathcal{L} x} \\ e_{\partial, \mathcal{L} x}\end{array}\right]$ and $y=\tilde{W}\left[\begin{array}{l}f_{\partial, \mathcal{L} x} \\ e_{\partial, \mathcal{L} x}\end{array}\right]$ where,

$$
\begin{aligned}
W & =\left[\begin{array}{cccccccc}
1 & 0 & 0 & 0 & 0 & 1 & 0 & 0 \\
0 & 0 & 1 & 0 & 0 & 0 & 0 & 1 \\
0 & -1 & 0 & 0 & 1 & 0 & 0 & 0 \\
0 & 0 & 0 & -1 & 0 & 0 & 1 & 0
\end{array}\right], \\
\tilde{W} & =\left[\begin{array}{ccccccccc}
0 & 1 & 0 & 0 & 1 & 0 & 0 & 0 \\
0 & 0 & 0 & 1 & 0 & 0 & 1 & 0 \\
1 & 0 & 0 & 0 & 0 & -1 & 0 & 0 \\
0 & 0 & 1 & 0 & 0 & 0 & 0 & -1
\end{array}\right] .
\end{aligned}
$$

It can by shown that with this choice of input and output the system (26) defines a an abstract boundary control system. Furthermore $\mathcal{A} x=P_{1}(\partial / \partial z)(\mathcal{L} x)+P_{0} \mathcal{L} x$ with domain $D(\mathcal{A})=$ $\left\{\mathcal{L} x \in H^{1}\left(a, b ; \mathbb{R}^{n}\right) \mid\left[\begin{array}{c}f_{\partial, \mathcal{L} x} \\ e_{\partial}, \mathcal{L} x\end{array}\right] \in \operatorname{ker} W\right\}$ generates a contraction semigroup on $X$ and the energy balance equation is defined as: $\frac{d E}{d t}=u^{T} y$.

Remark V.1. In this case the Timoshenko beam is fully actuated and the input/output set is defined by: $\left[\begin{array}{l}u \\ y\end{array}\right]=\frac{1}{\sqrt{2}}\left[\begin{array}{c}W \\ W\end{array}\right]\left[\begin{array}{cc}P_{1} & -P_{1} \\ I & I\end{array}\right]\left[\begin{array}{c}\mathcal{L} x(b) \\ \mathcal{L} x(a)\end{array}\right]$ where $\left[W^{T} \widetilde{W}^{T}\right]^{T}$ and $P_{1}$ are full rank. Hence there exists an $\varepsilon$ such that $\|u\|^{2}+\|y\|^{2} \geq \varepsilon\|\mathcal{L} x(b)\|^{2}$, and the BCS defined by the Timoshenko beam satisfies Assumption 2. 


\section{B. The DNA-bundle}

The DNA-bundle is represented by a simple damper + load + spring-damper system. In Figure $2, k_{2}, f_{1}, f_{2}, k_{\theta, 1}, k_{\theta, 2}, f_{\theta, 1}$, $f_{\theta, 2}$ represent the positive constants of the springs and the viscous dampers respectively, $M$ and $J$ are the mass and the inertia of the load and $x_{c 1}, x_{c 2}, \theta_{c 1}, \theta_{c 2}$ the relative positions and position angle respectively. Let us denote with sub-index $b$ the system representing the DNA-bundle. Then by defining $v_{b}=\left(x_{c 2}, M \dot{x}_{c 2}, \theta_{c 2}, J \dot{\theta}_{c 2}\right)^{T}$, $u_{b}=\left[\begin{array}{ll}F(b) & T(b)\end{array}\right]^{T}$ and $y_{b}=\left[\begin{array}{ll}v(b) & \omega(b)\end{array}\right]^{T}$ we obtain the following finite dimensional representation of the DNA-bundle

$$
\dot{v}_{b}=\left(J_{b}-R_{b}\right) \frac{d E_{b}}{d v_{b}}+g_{b} u_{b}, \quad y_{b}=g_{b}^{T} \frac{d E_{b}}{d v_{b}}+S_{b} u_{b}
$$

with $E_{b}$ the energy of the system (sum of the kinetic and potential energies): $E_{b}\left(x_{c 2}, M \dot{x}_{c 2}, \theta_{c 2}, J \dot{\theta}_{c 2}\right)=\frac{k_{2}}{2} x_{c 2}^{2}+\frac{1}{2 M}\left(M \dot{x}_{c 2}\right)^{2}+$ $\frac{k_{\theta, 2}}{2} \theta_{c 2}^{2}+\frac{1}{2 J}\left(J \dot{\theta}_{c 2}\right)^{2}$ and $J_{b}=\left[\begin{array}{ccccc}0 & 1 & 0 & 0 \\ -1 & 0 & 0 & 0 \\ 0 & 0 & 0 & 1 \\ 0 & 0 & -1 & 0\end{array}\right], R_{b}=\left[\begin{array}{cccc}0 & 0 & 0 & 0 \\ 0 & f_{2} & 0 & 0 \\ 0 & 0 & 0 & 0 \\ 0 & 0 & 0 & 0 \\ 0 & f_{\theta, 2}\end{array}\right]$, $g_{b}^{T}=\left[\begin{array}{llll}0 & 1 & 0 & 0 \\ 0 & 0 & 0 & 1\end{array}\right], S_{b}=\left[\begin{array}{cc}\frac{1}{f_{1}} & 0 \\ 0 & \frac{1}{f_{\theta, 1}}\end{array}\right]$. Note that this system satisfies Proposition III.1.

\section{The controller}

The controller, which we denote with the sub-index "a", is located at the base of the flexible arm, and is free to design. Hence the controller should be selected such that the conditions in Theorem IV. 2 are satisfied. To this end we chose the control system as

$$
\dot{v}_{a}=\left(J_{a}-R_{a}\right) \frac{d E_{a}}{d v_{a}}+g_{a} u_{a}, \quad y_{a}=g_{a}^{\top} \frac{d E_{a}}{d v_{a}}+S_{a} u_{a},
$$

where $v_{a}=\left[q_{a_{1}}, q_{a_{2}}, p_{a_{1}}, p_{a_{2}}\right]^{\top}, q_{a_{1}}, q_{a_{2}}$ are the generalized

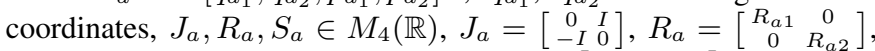
$S_{a}=\left[\begin{array}{cc}S_{a 1} & 0 \\ 0 & S_{a 2}\end{array}\right], g_{a}=\left[\begin{array}{l}0 \\ I\end{array}\right]$ with $R_{a i}=\left[\begin{array}{cc}r_{a_{i 1}} & 0 \\ 0 & r_{a_{i 2}}\end{array}\right] \in M_{2}(\mathbb{R})$, $r_{a_{i 1}}, r_{a_{i 2}}>0 \in \mathbb{R}, S_{a i}=\left[\begin{array}{cc}S_{a_{i 1}} & 0 \\ 0 & s_{a_{i 2}}\end{array}\right] \in M_{2}(\mathbb{R}), s_{a_{i 1}}, s_{a_{i 2}}>$ $0 \in \mathbb{R}, i=1,2$. The Hamiltonian of the system is given by the virtual energy: $E_{a}=\frac{1}{2}\left(k_{a_{1}} q_{a_{1}}^{2}+k_{a_{2}} q_{a_{2}}^{2}\right)+\frac{1}{2}\left(\frac{p_{a_{1}}^{2}}{m_{a}}+\frac{p_{a_{2}}^{2}}{m_{I_{a}}}\right)$ where $k_{a_{1}}, k_{a_{2}}, m_{a}, m_{I_{a}}$ are (positive) tuning parameters. The inputs $u_{a}=\left[u_{a_{1}}, u_{a_{2}}\right]^{\top} \in \mathbb{R}^{2}$ may be identified with the boundary variables of the beam at the point $a, u_{a}=[-v(a),-\omega(a)]^{\top}$. The outputs correspond to the input force and torque of the beam at point $a, y_{a}=[F(a), T(a)]^{\top}$. With this parametrization the finite dimensional port-Hamiltonian controller corresponds to an input strictly passive, exponentially stable system.

\section{Exponential stability}

The complete finite dimensional port-Hamiltonian control system is obtained by combining the port-Hamiltonian models of the DNAbundle and the controller,

$$
\begin{aligned}
\dot{v} & =\left[\begin{array}{c|c}
J_{b}-R_{b} & 0 \\
\hline 0 & J_{a}-R_{a}
\end{array}\right] \frac{d E_{c}}{d v}+\left[\begin{array}{c}
g_{b} \\
g_{a}
\end{array}\right] u_{c} \\
y_{c} & =\left[\begin{array}{ll}
g_{b}^{\top} & g_{a}^{\top}
\end{array}\right] \frac{d E_{c}}{d v}+\left[\begin{array}{c|c}
S_{a} & 0 \\
\hline 0 & S_{b}
\end{array}\right] u_{c}
\end{aligned}
$$

with state $v=\left[v_{a}, v_{b}\right]^{\top}$, Hamiltonian (storage) function $E_{c}=E_{a}+$ $E_{b}$, input $u_{c}=\left[u_{a}, u_{b}\right]^{\top}$, supply rate $w=u_{c}^{\top} y_{c}-u^{\top} S_{c} u$. The finite dimensional port-Hamiltonian system is strictly-input passive and exponentially stable, hence it satisfies Proposition III.1 and by Theorem IV.2 the microgripper i.e., the interconnection of the flexible arm, DNA-bundle and controller is exponentially stable.

\section{CONCLUSION}

The exponential stability of a class of BCS with dynamic boundary control has been addressed. The class of BCS encompasses infinite dimensional port-Hamiltonian systems and thereby a large number of physical systems. It has been shown that if the dynamic boundary controller is linear and input-strictly passive, then a power preserving interconnection renders the closed-loop system exponentially stable. The exponential stability follows from two key results: The power preserving interconnection between the class of BCS of Theorem II.1 and a strictly-input passive linear finite dimensional system defines again a BCS on an extended space; and, the dissipation of the controller assures the exponential decay of the total energy. The result is particularly interesting for stabilization of boundary control systems and for the analysis of complex systems whose models are given by coupled PDE's and ODE's. The result has been illustrated on a physically motivated example: a DNA manipulation process modelled by a PDE interconnected at the boundaries with a set of ODE's. Future work will aim to extend the results to energy shaping boundary controllers using Casimir's or/and IDA-PBC [14] techniques.

\section{REFERENCES}

[1] H. Fattorini, "Boundary control systems," SIAM Journal on Control, vol. 6, no. 3, pp. 349-385, 1968.

[2] R. Curtain and H. Zwart, An introduction to infinite-dimensional linear systems theory, ser. Texts in applied mathematics. New York, USA: Springer-Verlag, 1995.

[3] Z. Luo, B. Guo, and O.Morgul, Stability and Stabilization of Infinite Dimensional Systems with Applications, ser. Communication and control engineering. London, Great Britain: Springer-Verlag, 1999.

[4] M. Tucsnak and G. Weiss, Observation and Control for Operator Semigroups, ser. Birkhäuser Advanced Texts. Basel, Switzerland: Birkhäuser Verlag, 2009.

[5] Y. Le Gorrec, H. Zwart, and B. Maschke, "Dirac structures and boundary control systems associated with skew-symmetric differential operators," SIAM Journal on Control and Optimization, vol. 44, no. 5, pp. 18641892, 2005.

[6] J. Villegas, H. Zwart, Y. Le Gorrec, B. Maschke, and A. van der Schaft, "Stability and stabilization of a class of boundary control systems," in Decision and Control, 2005 and 2005 European Control Conference. CDC-ECC '05. 44th IEEE Conference on, dec. 2005, pp. 3850-3855.

[7] J. Villegas, H. Zwart, Y. Le Gorrec, and B. Maschke, "Exponential stability of a class of boundary control systems," Automatic Control, IEEE Transactions on, vol. 54, pp. 142-147, 2009.

[8] J. A. Villegas, "A port-Hamiltonian approach to distributed parameter systems," Ph.D. dissertation, Universiteit Twente, 2007.

[9] B. Jacob and H. Zwart, Linear Port-Hamiltonian Systems on Infinitedimensional Spaces, ser. Operator Theory: Advances and Applications. Basel, Switzerland: Birkhäuser, 2012, vol. 223.

[10] K. Yosida, Functional Analysis, ser. Classics in Mathematics. Berlin, Germany: Springer-Verlag, 1995

[11] J. Wen, "Time domain and frequency domain conditions for strict positive realness," Automatic Control, IEEE Transactions on, vol. 33, no. 10 , pp. 988-992, 1988.

[12] B. Brogliato, R. Lozano, B. Maschke, and O. Egeland, Dissipative Systems Analysis and Control, 2nd ed., ser. Communications and Control Engineering Series. London: Springer Verlag, 2007.

[13] J. Willems, "Dissipative dynamical systems part I: General theory," Archive for Rational Mechanics and Analysis, vol. 45, pp. 321-351, 1972.

[14] A. J. van der Schaft, L2-Gain and Passivity Techniques in Nonlinear Control, 2nd ed. New York, USA: Springer-Verlag, 2000.

[15] M. Boudaoud, Y. Haddab, and Y. Le Gorrec, "Modeling and optimal force control of a nonlinear electrostatic microgripper," Mechatronics, IEEE/ASME Transactions on, vol. 18, no. 3, pp. 1130-1139, June 2013.

[16] A. Macchelli and C. Melchiorri, "Modeling and control of the Timoshenko beam. the distributed port Hamiltonian approach," SIAM Journal on Control and Optimization, vol. 43, no. 2, pp. 743-767, 2004. 